\title{
openheart Prevalence of early stages of heart failure in an elderly risk population: the Copenhagen Heart Failure Risk Study
}

Freja Stoltze Gaborit, ${ }^{1,2}$ Caroline Kistorp, ${ }^{3,4}$ Thomas Kümler, ${ }^{1}$ Christian Hassager, ${ }^{3,5}$ Niels Tønder, ${ }^{6}$ Lars Køber, ${ }^{3,5}$ Pernille Mørk Hansen, ${ }^{7}$ Pia Rørbæk Kamstrup, ${ }^{8}$ Jens Faber, ${ }^{3,9}$ Kasper Karmark Iversen, ${ }^{1,3}$ Morten Schou ${ }^{1,3}$

\begin{abstract}
- Additional material is published online only. To view please visit the journal online (http://dx.doi.org/10.1136/ openhrt-2018-000840).
\end{abstract}

To cite: Gaborit FS, Kistorp C, Kümler T, et al. Prevalence of early stages of heart failure in an elderly risk population: the Copenhagen Heart Failure Risk Study. Open Heart 2019;6:e000840. doi:10.1136/ openhrt-2018-000840

Preliminary data from this study has been presented at the moderated poster session at the ESC HF 2018 Congress, Vienna, Austria.

Received 19 April 2018 Revised 31 October 2018 Accepted 9 December 2018

Check for updates

(C) Author(s) (or their employer(s)) 2019. Re-use permitted under CC BY-NC. No commercial re-use. See rights and permissions. Published by BMJ.

For numbered affiliations see end of article.

Correspondence to Dr Freja Stoltze Gaborit; freja. stoltze.gaborit@regionh.dk

\section{ABSTRACT}

Aims To describe the prevalence of heart failure (HF) stages in elderly outpatients with risk factors for HF but without known HF, and characterise the clinical, biochemical and echocardiographic findings in each stage. Background Early stages of HF are underdiagnosed; nevertheless, they are associated with an increased risk of hospitalisation and increased mortality. The prevalence of $\mathrm{HF}$ stages in elderly high-risk patients is unknown. Methods A total of 400 patients were consecutively included: $\geq 60$ years old, $\geq 1$ risk factor for HF (diabetes, chronic kidney disease, cardiovascular disease, atrial fibrillation, hypertension), and without known or suspected HF. HF stages were defined as the following: stage A (risk factor for HF, normal echocardiography), stage B (abnormal echocardiography, without symptoms of HF) and stage $\mathrm{C}$ (abnormal echocardiography, symptoms of $\mathrm{HF}$ clinical signs/increased plasma aminoterminal pro-B-type natriuretic peptide [NT-proBNP] concentrations).

Results After thorough examination $44.25 \%$ of patients were categorised as HF stage A, $37.5 \%$ were HF stage B and $18.25 \% \mathrm{HF}$ stage $\mathrm{C}$. Those with higher stages of HF were older $(p<0.001)$ and more often had atrial fibrillation $(p=0.006)$. The median plasma NT-proBNP concentrations $(\mathrm{pg} / \mathrm{mL})$ were 132.5 for HF stage A, 275.5 for HF stage $B$ and 400.0 for HF stage $C(p<0.001)$. Detectable plasma troponin-I was more frequent with abnormal echocardiography: HF stage A 9.7\%, HF stage B 27.3\% and HF stage $C 30.1 \%(p<0.001)$. HF stage $C$ reported higher score on the Minnesota Living with Heart Failure Questionnaire $(p<0.001)$.

Conclusions In an elderly high-risk population without known or suspected HF, more than half were HF stage $\mathrm{B}$ or $\mathrm{C}$. Higher stages of HF had increased plasma concentrations of NT-proBNP and troponin-I, besides a reduced quality of life. Focus on symptoms and signs of HF in this population are warranted.

\section{INTRODUCTION}

A large proportion of elderly people have early stages of heart failure (HF) based on the American College of Cardiology and the American Heart Association definition of HF stages. ${ }^{1}$ Increasing age of the general population and increasing prevalence of risk

\section{Key questions}

What is already known about this subject?

- The heart failure (HF) staging definition introduced by the American College of Cardiology and the American Heart Association underlines the importance of recognition of asymptomatic patients with early stages of $\mathrm{HF}$.

- Previous population-based studies have demonstrated a high prevalence of early stages of $\mathrm{HF}, \mathrm{HF}$ stages $A$ and $B$, with an increased risk of hospitalisation with $\mathrm{HF}$ and increased mortality.

What does this study add?

- In an elderly cohort living with risk factors for HF without known or suspected HF, more than half of the patients had an abnormal echocardiography and one-fifth had undiagnosed $\mathrm{HF}$.

- The most common type of HF in this cohort was HF with preserved ejection fraction (HFpEF).

- Our findings suggest that increased focus on HF symptoms and thorough examination of high-risk patients can identify undiagnosed $\mathrm{HF}$, in particular patients with HFpEF

- This should be considered in specialised outpatient clinics treating the risk factors for $\mathrm{HF}$ and at the department of cardiology.

How might this impact on clinical practice?

- Our results underscore the need for increased focus on HF symptoms in high-risk populations to improve management of early stages of HF and improve patients' quality of life, besides allocating patients with HFpEF to high-quality randomised clinical trials.

conditions (eg, hypertension, diabetes) lead to an increased number of persons with a risk of $\mathrm{HF}$, categorised as $\mathrm{HF}$ stage $\mathrm{A} .{ }^{23}$ Overt $\mathrm{HF}$, categorised as HF stage $\mathrm{C}$ or $\mathrm{D}$, is associated with poor prognosis, but also preclinical stages of HF, HF stage B, has an increased risk of hospitalisation with congestion and an increased mortality risk. ${ }^{4-7}$ Early recognition and improved treatment of risk conditions may delay the progression of early stages of HF. $^{8}$ Furthermore, promising research in 
the treatment of $\mathrm{HF}$ with preserved ejection fraction (HFpEF) is ongoing, and recognition of these patients needs to be improved to secure an optimal treatment.

Large community studies have observed that a considerable number of patients have early stages of HF. HF stage B was observed in 24\%-34\% and HF stage C in 12\%-13\% of these populations, with increasing prevalence with increasing age. ${ }^{4-6}$ The prevalence of HF stages B and C may, therefore, be even higher if a high-risk population is investigated, for example, elderly patients living with risk factors for HF.

The aim of this study was to evaluate the prevalence and clinical characteristics of HF stages in elderly patients with a high risk of HF, but without known or suspected HF.

\section{METHODS \\ Study population}

The Copenhagen Heart Failure Risk Study is a prospective cohort study. Patients were included from the Department of Cardiology, the Clinic of Diabetes and the Clinic of Nephrology at the Herlev and Gentofte University Hospital, Copenhagen, Denmark, enrolled from December 2014 to June 2016. Patients were screened consecutively during admission at the Department of Cardiology (screening 5 days a week), included from the day of discharge until 6 months later. Patients from the Clinic of Diabetes were screened consecutively by a treating physician at a scheduled visit (screening 2 days a week), included within 6 months. Patients from the Clinic of Nephrology were screened consecutively from the list of scheduled visits (screening 2 days a week), included within 6 months.

Inclusion and exclusion criteria are presented in Box 1. Data were collected during a single visit. Physical examination, medical history including current symptoms and medication, height, weight, non-invasive blood pressure, and 12-lead ECG were obtained; venous blood samples and spot-urinary sample were collected; patients fulfilled the Minnesota Living with Heart Failure Questionnaire (MLHFQ); and advanced echocardiography was performed. The Framingham probability of congestive HF within 4 years score was calculated. This is a risk prediction model that includes age, systolic blood pressure, heart rate, left ventricle (LV) hypertrophy on ECG, coronary heart disease, valve disease, diabetes and body mass index (if female) ${ }^{9}$

In total 3359 patients were screened and 400 patients were enrolled; inclusion flow is shown in figure 1 . The primary reason for patients not being eligible for this study was due to the presence of one or more exclusion criteria; especially patients from the Department of Cardiology often had advanced/ongoing cardiological diseases. No patients were excluded after inclusion, a full echocardiography was missing in one patient $(n=1)$, and plasma concentrations of aminoterminal pro-B-type
Box 1 Inclusion and exclusion criteria for the Copenhagen Heart Failure Risk Study

Inclusion criteria.

- Age $\geq 60$ years.

- Plus, one or more risk factor for heart failure (HF).

- Stable ischaemic heart disease.

- Peripheral arterial disease.

- Hypertension.

- History of atrial fibrillation/atrial flutter, diabetes.

- Apoplexia cerebri.

- Chronic kidney disease.

\section{Exclusion criteria.}

- Age $<60$ years.

- No risk factor of HF.

- Previously diagnosed with or admitted for HF, known left ventricle ejection fraction $\leq 45 \%$.

- Current admission with congestion or dyspnoea and suspected HF by the treating clinician.

- Advanced cardiac disease.

- Moderate-severe valvular disease, third-degree atrioventricular block, pacemaker with $>70 \%$ ventricular pace.

- Ongoing or recent cardiac disease or procedure.

- Cardioversion within the previous 30 days, coronary angiography or percutaneous coronary intervention or coronary artery bypass operation within the previous 30 days, ongoing endocarditis or myocarditis, or pulmonary embolism.

- Dialysis/estimated glomerular filtration rate $<15 \mathrm{~mL} / \mathrm{min} / 1.73 \mathrm{~m}^{2}$.

- Moderate-severe chronic obstructive pulmonary disease (global initiative for chronic obstructive lung disease stadium (GOLD) $\geq 2 \mathrm{C}$ ).

- Sepsis (systemic inflammatory response system criteria) within the previous 30 days or ongoing intravenous antibiotic.

- Estimated survival less than 1 year (eg, terminal cancer or due to frailty).

natriuretic peptide (NT-proBNP) was missing in one patient $(\mathrm{n}=1)$.

\section{Echocardiography}

Echocardiography was performed according to current guidelines. ${ }^{10}{ }^{11}$ Examinations were performed on Vivid E9 (General Electric Vingmed Ultrasound, Horten, Norway) by the same trained physician. Analyses were made off-line in EchoPAC (V.201,70.1, General Electric Vingmed Ultrasound) by one physician, blinded to clinical and biochemical outcomes. Before examination patients were registered by name and social security number, height and weight were entered, and a threelead ECG of good quality was continually registered. In patients with sinus rhythm, three loops were recorded, and for atrial fibrillation (AF) five loops were recorded. Images for strain should have a frame rate of $40-80$ frames/s, while images for tissue Doppler $>160$ frames/s.

We predefined a study definition of an 'abnormal echocardiography' based on current recommendations and previous studies in the elderly patients (online supplementary table 1). Abnormal structure of the LV was defined as LV end-diastolic volume (LVEDV) indexed to body surface area (BSA) (female: $>61 \mathrm{~mL} / \mathrm{m}^{2}$, male: $>74$ 


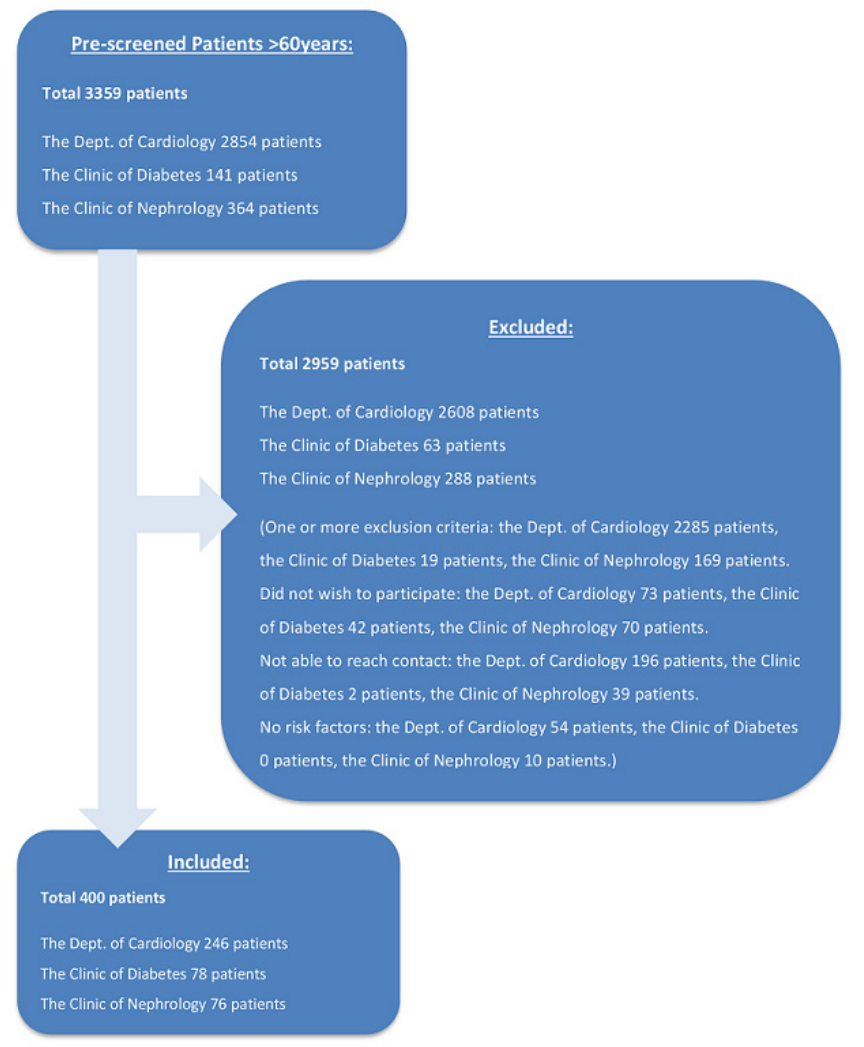

Figure 1 Inclusion flow of the Copenhagen Heart Failure Risk Study.

$\left.\mathrm{mL} / \mathrm{m}^{2}\right)^{10}$ and LV mass indexed to BSA (female: $>96 \mathrm{~g}$ / $\mathrm{m}^{2}$, male: $\left.>116 \mathrm{~g} / \mathrm{m}^{2}\right) \cdot{ }^{10}$ Abnormal systolic function was evaluated with left ventricle ejection fraction (LVEF) and global longitudinal strain (GLS). The limit for LVEF was set to detect mildly abnormal changes (female: $\leq 53 \%$, male: $\leq 51 \%$ ). The ARIC study has recently evaluated GLS in the HF staging system and confirmed its prognostic value for new-onset $\mathrm{HF}$ and mortality. ${ }^{4}$ However, validated reference limits do not exist, and therefore we used age-based and sex-based reference limits from the Framingham Heart Study ${ }^{12}$ (female: $\geq-15.3 \%$, male: $\geq-14.7 \%$ ).

\section{HF stages after examination}

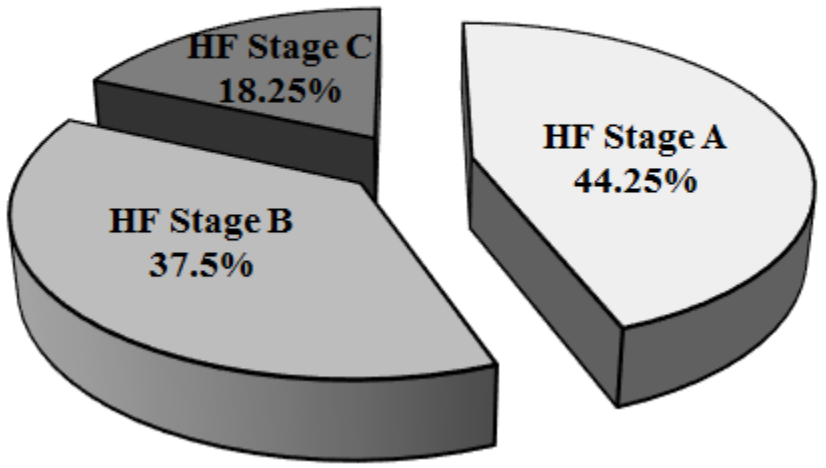

Figure 2 Prevalence of heart failure (HF) stages after clinical, biochemical and echocardiographic examination.
We included abnormal diastolic measures (tissue Doppler imaging and left atrial volume index), since these parameters have been associated to an increased risk of new-onset HF and mortality. ${ }^{45}$ The myocardial peak early velocity (e') is more affected by ageing, and we adjusted the study reference value according to normal values reported from the Atherosclerosis Risk in Communities Study $\left(\right.$ ARIC) ${ }^{4}$ (female: $<4.1 \mathrm{~cm} / \mathrm{s}$, male $<4.3 \mathrm{~cm} / \mathrm{s}$ ), while standard reference values were used for the more robust E/e' (lateral: $>13$, septal: $>15$ ) and for the left atrial volume indexed to BSA $\left(\geq 35 \mathrm{~mL} / \mathrm{m}^{2}\right) .{ }^{11}$ Significant valvular disease was defined as moderate-severe aortic stenosis (aortic valve area $<1 \mathrm{~cm}^{2}$ ), moderate-severe aortic regurgitation (visual judgement) and severe mitral stenosis (peak gradient mean $>12 \mathrm{~mm} \mathrm{Hg}$ ).

LVEF (\%) was calculated by the Simpson biplane method. Regional Wall Motion Score Index was calculated using the 16-segment model. LVEDV $(\mathrm{mL})$ was calculated from apical two-chamber and four-chamber views. $\mathrm{LV}$ mass index $\left(\mathrm{g} / \mathrm{m}^{2}\right)$ was calculated from linear dimensions in the parasternal view. Left atrial end-systolic volume $(\mathrm{mL})$ was calculated from apical two-chamber and four-chamber views. Myocardial peak early velocity $\left(e^{\prime}\right)(\mathrm{cm} / \mathrm{s})$ and peak systolic velocity $\left(\mathrm{s}^{\prime}\right)(\mathrm{cm} / \mathrm{s})$ were measured medially and septally at the mitral annulus with pulse wave tissue Doppler. Mitral inflow was measured with pulse wave Doppler, peak velocity of early filling (E) $(\mathrm{cm} / \mathrm{s})$ and atrial filling $(\mathrm{A})(\mathrm{cm} / \mathrm{s})$, and mitral valve deceleration time $(\mathrm{ms})$. E/e' ratio was calculated septally and laterally. Tricuspid annular plane systolic excursion $(\mathrm{mm})$ was measured with m-mode in the lateral tricuspid annulus. Longitudinal strain (\%) was measured in three apical views, and GLS was calculated as the average of the three, an 18-segment model. The presence of valvular heart disease was evaluated: aortic sclerosis by visual judgement, aortic stenosis calculated (aortic velocity time integral [VTI], LV outflow tract VTI and diameter), aortic regurgitation by visual judgement of colour flow, mitral stenosis calculated (mitral valve VTI mean gradient), and mitral regurgitation by visual judgement of colour flow.

\section{Definition of HF stages}

HF stages were defined according to the American College of Cardiology/American Heart Association definition ${ }^{1}$ :

- HF stage A: patients with risk factors of HF, with normal echocardiography and without signs or symptoms of HF.

- HF stage B: patients with risk factors of HF, with an abnormal echocardiography but without past or current symptoms of HF.

- HF stage C: patients with an abnormal echocardiography and past or current symptoms of HF.

To ensure the HF diagnoses in patients categorised as HF stage C, we used the European Society of Cardiology definition of HF. ${ }^{14}$ Signs of HF may be absent, for which reason a biochemical confirmation of $\mathrm{HF}$ was included. Patients in HF stage $\mathrm{C}$ needed to fulfil three criteria: 
(1) symptoms of HF (two of the following: dyspnoea or orthopnoea and oedema, or treatment with loop diuretics); (2) abnormal echocardiography according to study definition; and (3) clinical signs and/or abnormal plasma concentrations of NT-proBNP (sinus rhythm $>125$ $\mathrm{ng} / \mathrm{L},{ }^{14} \mathrm{AF}>524 \mathrm{pg} / \mathrm{mL}^{15}$ ).

\section{Biochemistry}

Venous blood samples were drawn on the day of examination. Routine blood samples (eg, haemoglobin, creatinine, highly sensitive $\mathrm{C}$ reactive peptide [hsCRP]) and urine-albumin were analysed successively. Blood samples for later tests were centrifuged at $4^{\circ}$, and plasma was stored at $-80^{\circ} \mathrm{C}$. Plasma concentrations of NT-proBNP (ng/L) were measured with the IMMULITE 2000 NT-proBNP, solid-phase, two-site chemiluminescent immunometric assay (Siemens Healthcare Diagnostics). ${ }^{16}$ Plasma concentrations of mid-regional proadrenomedullin (MR-proADM) (nmol/L) and copeptin (C-terminal provasopressin) $(\mathrm{pmol} / \mathrm{L})$ were measured on BRAHMS KRYPTOR with specified automated immunofluorescent assays. ${ }^{17} 18$ Plasma concentrations of troponin-I were measured with ADVIA Centaur TnI-Ultra assay (Siemens Healthcare Diagnostics). ${ }^{19}$ Concentrations of urinary albumin were measured in spot-urine. Microalbuminuria was defined as $20-199 \mathrm{mg} / \mathrm{L}$ and macroalbuminuria $\geq 200 \mathrm{mg} / \mathrm{L}$.

\section{Statistical methods}

Patients were categorised in HF stages A, B and C. Dichotomous variables were presented as numbers and prevalences (\%). Continuous variables were both normally and skewed distributed and are presented as medians with IQRs (25th and 75th percentiles). Baseline characteristics and echocardiographic parameters were compared across HF stages, with pairwise comparisons between groups. For continuous variables, we used Wilcoxon rank-sum test (Kruskal-Wallis test) and a trend test, and for dichotomous variables we used $\chi^{2}$ test. In the pairwise comparison, we adjusted for multiple testing with the Bonferroni correction. Skewed distributed variables were $\log$ 2-transformed when appropriate to reduce the effects of skewness. Trend tests were performed using the Cochran-Armitage trend test for proportions and logistic regression models for continuous variables (dependent variable: HF stages). Multivariate linear and logistic regression models were performed to evaluate the associations between the HF stages and baseline characteristic with a $p$ value $<0.05$ in the trend test. The regression models were adjusted for age, gender, AF, hypertension, ischaemic heart disease, stroke, diabetes, estimated glomerular filtration rate (eGFR) and department from where the patients were included. A two-sided $p$ value $<0.05$ was considered significant, and in case of Bonferroni correction due to multiple testing a $\mathrm{p}$ value $<0.05 / 3 \quad(=0.017)$ was considered significant. Analyses were performed using the SAS Enterprise Guide V.7.11 statistical software.

\section{Sample size}

It was anticipated that the prevalence of patients with $\mathrm{HF}$ stage B or C would be $30 \%$. With a power of $80 \%$ at a significance level of $5 \%$, a total of 369 patients should be included. To account for dropouts and eventually missing data, 400 patients were included.

\section{RESULTS}

\section{Prevalence of HF stages}

Before examination by default, all patients were considered as HF stage A, based on the inclusion and exclusion criteria. After examination 177 (44.25\%) remained as $\mathrm{HF}$ stage A, $150(37.5 \%)$ were HF stage B and 73 (18.25\%) were HF stage C, illustrated in figure 2. In HF stage A, $29.9 \%$ reported symptoms of HF, 36.7\% had clinical signs of $\mathrm{HF}$ and $50.6 \%$ had elevated plasma concentrations of NT-proBNP. However, these patients did not fulfil all the criteria for an HF diagnosis, and their symptoms may therefore be considered non-cardiac. Only $28.25 \%$ in HF stage A had no symptoms of HF, no clinical signs and normal plasma concentrations of NT-proBNP. In HF stage B, all patients had an abnormal echocardiography, $38.0 \%$ had clinical signs of $\mathrm{HF}$ and $73.3 \%$ had elevated plasma concentrations of NT-proBNP, and $30.7 \%$ had both clinical signs of $\mathrm{HF}$ and elevated plasma concentrations of NT-proBNP. Two patients did not fit with the definition of HF stages; they had an abnormal echocardiography and symptoms of HF, but no clinical signs nor elevated plasma concentrations of NT-proBNP. They were categorised as HF stage $\mathrm{B}$. Patients in HF stage $\mathrm{C}$ had an abnormal echocardiography and symptoms of HF; $78.1 \%$ had clinical signs of $\mathrm{HF}$ and $83.6 \%$ had elevated plasma concentrations of NT-proBNP, and $61.6 \%$ had both clinical signs of HF and elevated plasma concentrations of NT-proBNP.

\section{Characterisation of HF stages}

Baseline characteristics are presented in table 1. The median age was 72 years, ranging from 60 to 97 , and patients in HF stage $B$ and $C$ were older than patients in HF stage A $(p<0.001$ for trend). Of the patients, $48.5 \%$ were female, with no significant difference between stages of $\mathrm{HF}$.

Patients with $\mathrm{HF}$ stage $\mathrm{C}$ were more frequently admitted due to dyspnoea or palpitations. However, in all stages of $\mathrm{HF}$, chest pain was the dominating reason for hospitalisation, and it did not differ between the different stages. The frequencies of chest pain and palpitations were almost identical to the frequencies of ischaemic heart disease and AF (table 1).

There was no difference in 'numbers of risk factors' ( $\mathrm{p}=0.093$ for trend); overall 119 patients had one risk factor, 184 had two risk factors, 79 had three risk factors and 18 had more than four risk factors. The only risk factor that differed in prevalence was a history of AF, with more patients in higher stages of HF ( $p=0.001$ for trend). Patients with higher stages of HF had lower eGFR $(p=0.003$ for trend $)$, haemoglobin $(p=0.015$ for trend $)$ 
Heart failure and cardiomyopathies

Table 1 Clinical characteristics of patients in the Copenhagen Heart Failure Risk Study, according to HF stage

\begin{tabular}{|c|c|c|c|c|c|}
\hline & $\begin{array}{l}\text { All patients } \\
(n=400)\end{array}$ & $\begin{array}{l}\text { HF stage A } \\
(n=177)\end{array}$ & $\begin{array}{l}\text { HF stage B } \\
(n=150)\end{array}$ & $\begin{array}{l}\text { HF stage C } \\
(n=73)\end{array}$ & $\begin{array}{l}P \text { value for } \\
\text { trend }\end{array}$ \\
\hline Age, years & $72(67,78)$ & $69(65,73)^{*} \dagger$ & $75(69,79)$ & $74(69,82)$ & $<0.001$ \\
\hline Female sex, n (\%) & $194(48.5)$ & $78(44.1)$ & $74(49.3)$ & $42(57.5)$ & 0.053 \\
\hline $\mathrm{BMI}, \mathrm{kg} / \mathrm{m}^{2}$ & $27.3(24.5,30.9)$ & $27.1(24.3,30.7)$ & $27.2(24.5,30.1)$ & $28.3(24.7,32.8)$ & 0.117 \\
\hline $\mathrm{BMI} \geq 30, \mathrm{n}(\%)$ & $119(29.8)$ & $52(29.4)$ & $39(26.0)$ & $28(38.4)$ & 0.309 \\
\hline Systolic blood pressure, $\mathrm{mm} \mathrm{Hg}$ & $137.0(126.5,149.0)$ & $134.0(126.0,146.0)$ & $140.0(128.0,150.0)$ & $137(125.0,150.0)$ & 0.312 \\
\hline Diastolic blood pressure, $\mathrm{mm} \mathrm{Hg}$ & $79.0(72.0,85.0)$ & $81.0(74.0,85.0) \dagger$ & $78.0(71.0,86.0)$ & $77.0(69.0,84.0)$ & 0.0135 \\
\hline $\mathrm{HR}$, beats/min & $69.0(60.0,79.0)$ & $69.0(61.0,78.0)$ & $68.5(60.0,81.0)$ & $69.0(60.0,79.0)$ & 0.471 \\
\hline Left branch bundle block ECG, n (\%) & $13(3.3)$ & $1(0.6)^{\star} \dagger$ & $8(5.3)$ & $4(5.6)$ & 0.015 \\
\hline Right branch bundle block ECG, $n(\%)$ & $25(6.3)$ & $11(6.2)$ & $7(4.7)$ & $7(9.7)$ & 0.475 \\
\hline LV hypertrophy ECG, n (\%) & $7(1.8)$ & $2(1.1)$ & $4(2.7)$ & $1(1.4)$ & 0.675 \\
\hline NYHA class I, n (\%) & $235(58.8)$ & $117(66.1) \dagger$ & $98(65.3) \ddagger$ & $20(27.4)$ & $<0.001$ \\
\hline NYHA class II, $\mathrm{n}(\%)$ & $145(36.3)$ & $55(31.1) \dagger$ & $45(30.0) \ddagger$ & $45(61.6)$ & $<0.001$ \\
\hline NYHA class III, n (\%) & $20(5.0)$ & $5(2.8) \dagger$ & $7(4.7)$ & $8(11.0)$ & 0.012 \\
\hline Smoking (former or active), n (\%) & $249(62.3)$ & $120(67.8)^{\star}$ & $81(54.0)$ & $48(65.8)$ & 0.316 \\
\hline \multicolumn{6}{|l|}{ Variables for HF staging } \\
\hline Symptoms of heart failure, $\mathrm{n}(\%)$ & $128(32.0)$ & $53(29.9)^{*} \dagger$ & $2(1.3) \ddagger$ & $73(100)$ & \\
\hline Clinical signs of heart failure, $\mathrm{n}(\%)$ & $179(44.8)$ & $65(36.7) \dagger$ & $57(38.0) \ddagger$ & $57(78.1)$ & \\
\hline Abnormal echocardiography, $\mathrm{n}(\%)$ & $223(55.8)$ & $0(0.0)^{*} \dagger$ & $150(100)$ & $73(100)$ & \\
\hline MLHFQ & $12.0(2.0,28.0)$ & $11.0(2.0,28.0) \dagger$ & $8.0(1.0,17.0) \ddagger$ & $25.0(16.0,42.0)$ & 0.001 \\
\hline Framingham 4-year HF risk score & $11.0(8.0,15.0)$ & $9.0(7.5,14.0)^{\star} \dagger$ & $11.5(9.0,16.0)$ & $12.0(9.0,16.0)$ & $<0.001$ \\
\hline Indication for hospitalisation & & & & & 0.043 \\
\hline Chest pain, $\mathrm{n}(\%)$ & $115(28.8)$ & $55(31.1)$ & $38(25.3)$ & $22(30.1)$ & 0.646 \\
\hline Palpitations/Arrhythmia, n (\%) & $80(20.0)$ & $25(14.1)$ & $36(24.0)$ & $19(26.0)$ & 0.013 \\
\hline Syncope, n (\%) & $30(7.5)$ & $11(6.2)$ & $13(8.7)$ & $6(8.2)$ & 0.477 \\
\hline Dyspnoea, n (\%) & $12(3.0)$ & $4(2.3)$ & $2(1.3)$ & $6(8.2)$ & 0.044 \\
\hline Other, n (\%) & $9(2.3)$ & $4(2.3)$ & $5(3.3)$ & $0(0)$ & 0.545 \\
\hline $\begin{array}{l}\text { Ambulatory visits-Clinic of Diabetes, } \\
\mathrm{n}(\%)\end{array}$ & 78 (19.5) & $40(22.6)$ & $28(18.7)$ & $10(13.7)$ & 0.100 \\
\hline $\begin{array}{l}\text { Ambulatory visits-Clinic of Nephrology, } \\
\mathrm{n}(\%)\end{array}$ & $76(19.0)$ & $38(21.5)$ & $28(18.7)$ & $10(13.7)$ & 0.160 \\
\hline \multicolumn{6}{|l|}{ Medical history } \\
\hline Hypertension, n (\%) & $328(82.0)$ & $145(81.9)$ & $120(80.0)$ & $63(86.3)$ & .568 \\
\hline Ischaemic heart disease, n (\%) & $98(24.5)$ & $37(20.9)$ & $41(27.3)$ & $20(27.4)$ & 0.187 \\
\hline Atrial fibrillation, $\mathrm{n}(\%)$ & $119(29.8)$ & $40(22.6) \dagger$ & $48(32.0)$ & $31(42.5)$ & 0.001 \\
\hline Diabetes, n (\%) & $143(35.8)$ & $69(39.0)$ & $51(34.0)$ & $23(31.5)$ & 0.218 \\
\hline Chronic kidney disease, n (\%) & $63(15.8)$ & $30(17.0)$ & $23(15.3)$ & $10(13.7)$ & 0.506 \\
\hline Apoplexia cerebri, n (\%) & $48(12.0)$ & $19(10.7)$ & $23(15.3)$ & $6(8.2)$ & 0.915 \\
\hline Mild COPD or asthma, $\mathrm{n}(\%)$ & $34(8.5)$ & $18(10.2)$ & $6(4.0) \ddagger$ & $10(13.7)$ & 0.840 \\
\hline \multicolumn{6}{|l|}{ Evaluation of risk factors } \\
\hline Risk factors, n (\%) & $2.0(1.0,2.0)$ & $2.0(1.0,2.0)$ & $2.0(1.0,3.0)$ & $2.0(2.0,2.0)$ & 0.093 \\
\hline More than two risk factors, $\mathrm{n}(\%)$ & $97(24.3)$ & $39(22.0)$ & $41(27.3)$ & $17(23.3)$ & 0.615 \\
\hline \multicolumn{6}{|l|}{ Medication } \\
\hline ACE inhibitor, n (\%) & $105(26.3)$ & $49(27.7)$ & $45(30.0) \ddagger$ & $11(15.1)$ & 0.103 \\
\hline Angiotensin receptor antagonist, $\mathrm{n}(\%)$ & $139(34.8)$ & $59(33.3)$ & $51(34.0)$ & $29(39.7)$ & 0.388 \\
\hline Aldosterone antagonist, $\mathrm{n}(\%)$ & $5(1.3)$ & $0(0.0)$ & $3(2.0)$ & $2(2.7)$ & 0.047 \\
\hline Calcium antagonist, n (\%) & $125(31.3)$ & $48(27.1)$ & $50(33.3)$ & $27(37.0)$ & 0.097 \\
\hline
\end{tabular}




\begin{tabular}{|c|c|c|c|c|c|}
\hline & $\begin{array}{l}\text { All patients } \\
(n=400)\end{array}$ & $\begin{array}{l}\text { HF stage A } \\
(n=177)\end{array}$ & $\begin{array}{l}\text { HF stage B } \\
(n=150)\end{array}$ & $\begin{array}{l}\text { HF stage C } \\
(n=73)\end{array}$ & $\begin{array}{l}P \text { value for } \\
\text { trend }\end{array}$ \\
\hline Loop diuretics, n (\%) & $60(15.0)$ & $16(9.0)^{\star}$ & $15(10.0) \ddagger$ & $29(39.7)$ & $<0.001$ \\
\hline Thiazide, n (\%) & $125(31.3)$ & $62(35.0) \dagger$ & 49 (32.7) & 14 (19.2) & 0.025 \\
\hline Insulin, n (\%) & $65(16.3)$ & 34 (19.2) & $22(14.7)$ & $9(12.3)$ & 0.141 \\
\hline \multicolumn{6}{|l|}{ Biochemistry } \\
\hline Haemoglobin, $g / L$ & $138.6(128.9,146.6)$ & $140.2(132.1,148.2) \dagger$ & $137.0(125.7,146.6)$ & $133.7(127.3,145.0)$ & 0.015 \\
\hline Thrombocytes, $\times 10^{9} / \mathrm{L}$ & $232.2(190.5,280.0)$ & $230.0(193.0,281.0)$ & $228.5(189.0,270.0)$ & $242.0(184.0,286.0)$ & 0.960 \\
\hline Creatinine, $\mu \mathrm{mol} / \mathrm{L}$ & $82.0(69.0,106.0)$ & $80.0(66.0,103.0)$ & $82.0(71.0,109.0)$ & $87.0(68.0,116.0)$ & 0.213 \\
\hline eGFR, $\mathrm{mL} / \mathrm{min} / 1.73 \mathrm{~m}^{2}$ & $70.0(53.0,86.0)$ & $76.0(57.5,90.0)^{*} \dagger$ & $65.5(51.0,84.0)$ & $65.0(47.5,83.5)$ & 0.003 \\
\hline Microalbuminuria, \% & $143(37.9)$ & $51(31.1)$ & $61(43.3)$ & $31(43.1)$ & 0.036 \\
\hline Macroalbuminuria, \% & $38(10.1)$ & $13(7.9)^{\star}$ & $18(12.8)$ & $7(9.7)$ & 0.457 \\
\hline $\mathrm{HbA} 1 \mathrm{c}, \%$ & $5.8(5.5,6.6)$ & $5.8(5.6,6.9)$ & $5.8(5.5,6.5)$ & $5.8(5.6,6.4)$ & 0.186 \\
\hline Albumin, $g / L$ & $42.0(40.0,44.0)$ & $43.0(40.0,45.0)^{\star} \dagger$ & $42.0(40.0,44.0)$ & $41.0(40.0,44.0)$ & $<0.001$ \\
\hline Bilirubin, $\mu \mathrm{mol} / \mathrm{L}$ & $10.0(8.0,14.0)$ & $10.0(7.0,14.0)$ & $11.0(8.0,14.0)$ & $10.0(8.0,14.5)$ & 0.037 \\
\hline ALAT, U/L & $29.0(22.0,39.0)$ & $31.0(24.0,39.0)$ & $27.0(20.0,40.0)$ & $26.0(20.0,37.0)$ & 0.524 \\
\hline TSH, $\times 10^{-3} \mathrm{IE} / \mathrm{L}$ & $1.3(0.8,1.9)$ & $1.3(0.8,1.8)$ & $1.2(0.8,1.8)$ & $1.6(0.9,2.4)$ & 0.109 \\
\hline MR-proADM, nmol/L & $0.85(0.66,1.06)$ & $0.75(0.59,0.95)^{\star} \dagger$ & $0.87(0.69,1.07) \ddagger$ & $1.00(0.84,1.17)$ & $<0.001$ \\
\hline Copeptin, pmol/L & $7.2(4.5,14.3)$ & $6.4(3.8,12.5)$ & $7.6(4.9,14.5)$ & $9.3(5.2,19.7)$ & 0.013 \\
\hline
\end{tabular}

Categorical values are presented in percentages, and continuous values are presented as median with 25th and 75th percentile.

${ }^{*} \mathrm{P}<0.017$ for HF stage A vs HF stage $B$.

$\dagger \mathrm{P}<0.017$ for HF stage A vs HF stage $\mathrm{C}$.

$\ddagger \mathrm{P}<0.017$ for $\mathrm{HF}$ stage $\mathrm{B}$ vs HF stage $\mathrm{C}$.

ALAT, alanine transaminase; BMI, body mass index; COPD, chronic obstructive pulmonary disease; eGFR, estimated glomerular filtration rate; $\mathrm{HbA1c}$, haemoglobin $\mathrm{A1c}$; $\mathrm{HDL}$, high-density lipoprotein; $\mathrm{HF}$, heart failure; $\mathrm{HR}$, heart rate; hsCRP, highly sensitive $\mathrm{C}$ reactive peptide; LDL, low-density lipoprotein; LV, left ventricle; MLHFQ, Minnesota Living with Heart Failure Questionnaire; MR-proADM, mid-regional proadrenomedullin; NT-proBNP, aminoterminal pro-brain natriuretic peptide; NYHA, New York Heart Association; TSH, thyroid stimulating hormone.

and plasma albumin $(\mathrm{p}<0.001$ for trend) (table 1$)$. In the adjusted analyses the associations between HF stages and age, diastolic blood pressure, MLHFQ, haemoglobin, eGFR, prevalence of microalbuminuria and bilirubin remained significant (table 2).

Plasma concentrations of NT-proBNP increased with higher stages of HF $(p<0.001)$, with a significant difference between each stage, illustrated in figure 3. Plasma concentrations of troponin-I were more frequently increased in patients with HF stages B and C ( $<<0.001$ for trend). Plasma concentrations of copeptin and MR-proADM also increased with higher stages of $\mathrm{HF}$ ( $\mathrm{p}<0.001$ for trend). MLHFQ score was higher in patients with HF stage C, reflecting a decreased quality of life $(\mathrm{p}<0.001$ for trend). Framingham probability of congestive HF within 4 years score was higher in patients with HF stages $B$ and $C(p<0.001$ for trend), illustrated in figure 3. In the adjusted analyses the associations between HF stages and increased plasma troponin-I concentrations $(\mathrm{p}=0.010)$, plasma NT-proBNP concentrations $(p<0.001)$, plasma MR-proADM concentrations $(p<0.001)$ and plasma copeptin concentrations $(p=0.009)$ remained significant (table 2$)$. 
Table 2 Multivariate regression models according to HF stages

Multivariate linear regression models according to HF stages

\begin{tabular}{|c|c|c|c|}
\hline & Estimate & $95 \% \mathrm{Cl}$ & $P$ value \\
\hline Age & & & $<0.001$ \\
\hline HF stage $A$ & Ref & & \\
\hline HF stage B & 3.55 & 2.137 to 4.956 & $<0.001$ \\
\hline HF stage $\mathrm{C}$ & 3.33 & 1.525 to 5.128 & $<0.001$ \\
\hline Diastolic blood pressure & & & $<0.001$ \\
\hline HF stage $A$ & Ref & & \\
\hline HF stage B & -0.09 & -2.291 to 2.106 & 0.934 \\
\hline HF stage $\mathrm{C}$ & -1.87 & -4.640 to 0.901 & 0.185 \\
\hline $\begin{array}{l}\text { Minnesota Living } \\
\text { with Heart Failure } \\
\text { Questionnaire }\end{array}$ & & & 0.049 \\
\hline HF stage $A$ & Ref & & \\
\hline HF stage B & -6.11 & -10.32 to 1.89 & 0.005 \\
\hline HF stage $\mathrm{C}$ & 12.33 & 7.18 to 17.56 & $<0.001$ \\
\hline $\begin{array}{l}\text { Framingham 4-year risk } \\
\text { of congestion }\end{array}$ & & & 0.118 \\
\hline HF stage $A$ & Ref & & \\
\hline HF stage B & 0.36 & -0.33 to 1.05 & 0.306 \\
\hline HF stage $\mathrm{C}$ & 0.61 & -0.26 to 1.48 & 0.17 \\
\hline Haemoglobin & & & $<0.001$ \\
\hline HF stage $A$ & Ref & & \\
\hline HF stage B & -0.06 & -0.26 to 0.14 & 0.528 \\
\hline HF stage $\mathrm{C}$ & -0.15 & -0.41 to 0.10 & 0.232 \\
\hline eGFR & & & $<0.001$ \\
\hline HF stage $A$ & Ref & & \\
\hline HF stage B & -1.36 & -5.25 to 2.54 & 0.495 \\
\hline HF stage $\mathrm{C}$ & -2.77 & -7.67 to 2.14 & 0.269 \\
\hline Bilirubin & & & $<0.001$ \\
\hline HF stage $A$ & Ref & & \\
\hline HF stage B & 1.04 & -0.08 to 2.17 & 0.069 \\
\hline HF stage $\mathrm{C}$ & 1.1 & -0.32 to 2.52 & 0.128 \\
\hline Albumin & & & $<0.001$ \\
\hline HF stage A & Ref & & \\
\hline HF stage B & -0.65 & -1.39 to 0.09 & 0.084 \\
\hline HF stage $\mathrm{C}$ & -0.84 & -1.76 to 0.09 & 0.077 \\
\hline NT-proBNP & & & $<0.001$ \\
\hline HF stage $A$ & Ref & & \\
\hline HF stage B & 0.34 & 0.02 to 0.66 & 0.036 \\
\hline HF stage $\mathrm{C}$ & 0.87 & 0.48 to 1.28 & $<0.001$ \\
\hline MR-proADM & & & $<0.001$ \\
\hline HF stage $A$ & Ref & & \\
\hline HF stage B & 0.07 & -0.03 to 0.17 & 0.179 \\
\hline HF stage $\mathrm{C}$ & 0.29 & 0.16 to 0.42 & $<0.001$ \\
\hline
\end{tabular}

Continued
Table 2 Continued

Multivariate linear regression models according to HF stages

\begin{tabular}{clcc}
\hline & Estimate & $\mathbf{9 5 \%} \mathbf{C l}$ & P value \\
\hline Copeptin & & & 0.009 \\
\hline HF stage A & Ref & & \\
HF stage B & 0.16 & -0.15 to 0.35 & 0.422 \\
HF stage C & 0.16 & 0.11 to 0.74 & 0.008 \\
\hline
\end{tabular}

Multivariate logistic regression models according to HF stages

\begin{tabular}{lllc}
\hline & OR & 95\% Cl & P value \\
\hline $\begin{array}{c}\text { Left branch bundle block on } \\
\text { ECG }\end{array}$ & & 0.217 \\
\hline HF stage A vs HF stage B & 6.44 & 0.68 to 60.56 & 0.104 \\
\hline HF stage A vs HF stage C & 8.16 & 0.74 to 90.51 & 0.087 \\
\hline NYHA class & & & \\
\hline HF stage A vs HF stage B & 1.21 & 0.74 to 1.99 & 0.446 \\
\hline HF stage A vs HF stage C & 0.29 & 0.16 to 0.52 & $<0.001$ \\
\hline Atrial fibrillation & & & $<0.001$ \\
\hline HF stage A vs HF stage B & 1.48 & 0.78 to 2.80 & 0.227 \\
\hline HF stage A vs HF stage C & 2.39 & 1.12 to 5.10 & 0.024 \\
\hline Microalbuminuria & & & 0.008 \\
\hline HF stage A vs HF stage B & 1.35 & 0.82 to 2.25 & 0.241 \\
\hline HF stage A vs HF stage C & 1.3 & 0.69 to 2.44 & 0.412 \\
\hline Troponin-I $\geq 10$ ng/L & & & 0.01 \\
\hline HF stage A vs HF stage B & 2.35 & 1.21 to 4.54 & 0.011 \\
\hline HF stage A vs HF stage C & 2.55 & 1.18 to 5.50 & 0.017 \\
\hline
\end{tabular}

The response variables are specified below. The predictor variables are HF stages, age, gender, atrial fibrillation, hypertension, ischaemic heart disease, stroke, diabetes, kidney function (eGFR) and department from where the patients are included.

HF, heart failure; MR-proADM, mid-regional proadrenomedullin; NT-proBNP, aminoterminal pro-brain natriuretic peptide; NYHA, New York Heart Association; Ref, reference; eGFR, estimated glomerular filtration rate.

Echocardiographic parameters are presented in table 3. An 'abnormal echocardiography' was part of the HF stages $\mathrm{B}$ and $\mathrm{C}$ definition, and this reflects in the results with no major differences between the two. The only echocardiographic parameters with a difference between HF stages $B$ and $\mathrm{C}$ were the proportion of abnormal LV mass index ( $<<0.001$ for trend) and the proportion of abnormal e' septal ( $<<0.0001$ for trend). The median LVEF was $62.64 \%$, slightly lower in higher stages of HF $(p<0.0001)$. In total 12 patients $(3 \%)$ had an LVEF $\leq 40 \%$, of these 8 had no symptoms and were therefore HF stage $\mathrm{B}$, while 4 reported symptoms of HF and were therefore $\mathrm{HF}$ stage $\mathrm{C}$.

Despite having excluded patients with known moderate-severe valvular heart disease, two patients were encountered with moderate-severe aortic stenosis (aortic 


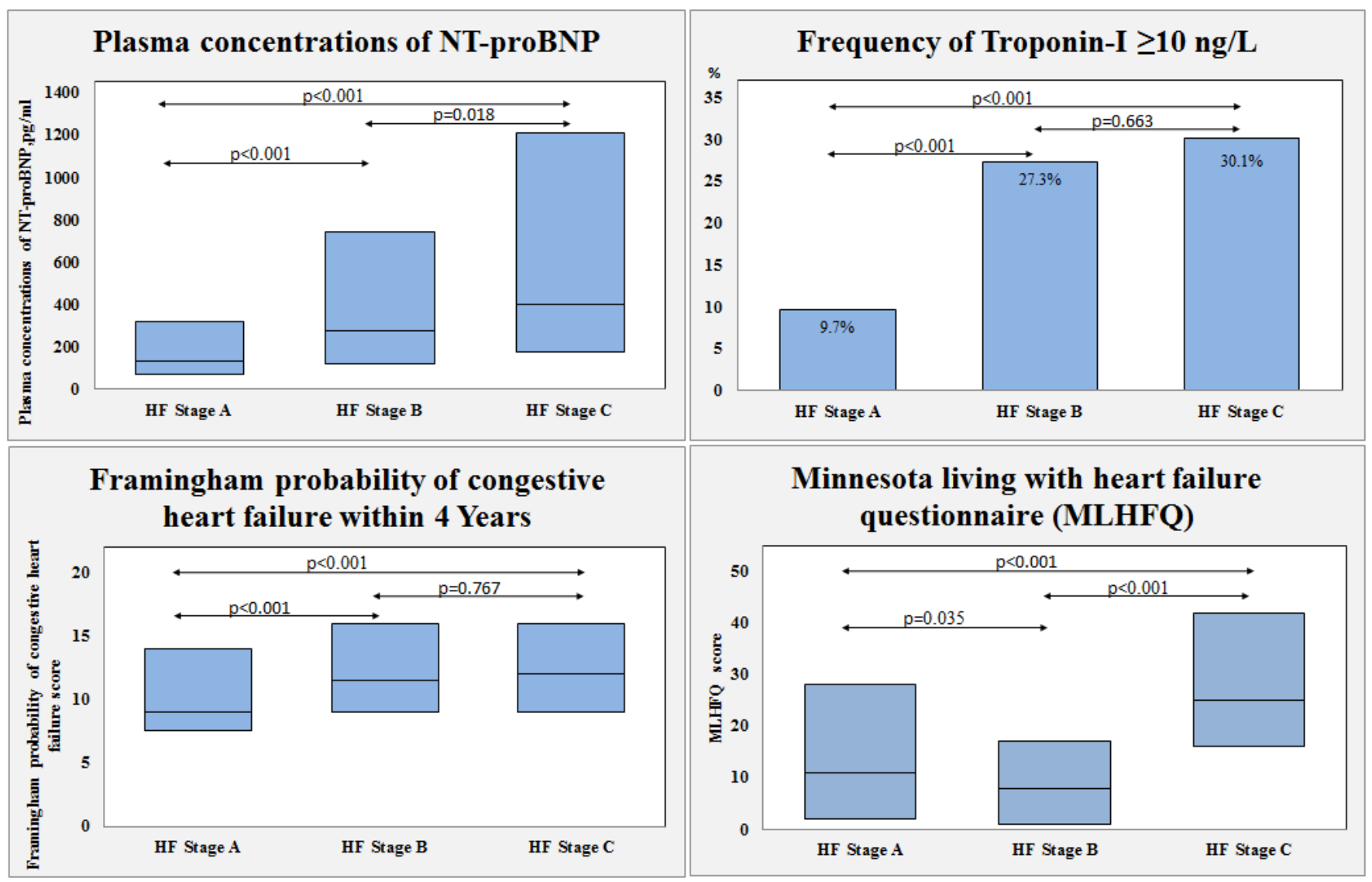

Figure 3 Cardiac biomarkers, quality of life and Framingham HF risk score according to HF stages. Plasma concentrations of NT-proBNP presented as median with 25th and 75th percentiles and patients with elevated troponin-I ( $\geq 10 \mathrm{ng} / \mathrm{L})$ as proportion (\%) according to HF stages. Minnesota Living with Heart Failure Questionnaire and Framingham probability of congestive HF within 4 years score presented as median with 25th and 75th percentiles. HF, heart failure; NT-proBNP, aminoterminal pro-brain natriuretic peptide.

valve area $<1.0 \mathrm{~cm}^{2}$ ) and one with moderate-severe aortic regurgitation. No patients with severe mitral regurgitation were encountered.

\section{DISCUSSION}

The main findings of this study were that HF stages B and $\mathrm{C}$ are frequent in an elderly high-risk population without known or suspected HF, and especially HFpEF is underdiagnosed. Clinical characteristics were similar in HF stages $\mathrm{B}$ and C; however, plasma concentrations of NT-proBNP increased with higher stages of HF, whereas troponin-I was more frequently detectable in patients with an abnormal echocardiography. Finally, higher stages of HF had decreased quality of life.

\section{Clinical characteristics of HF stages}

Elderly patients with one or more risk factor for HF often present with symptoms compatible with HF. After a thorough examination, we observed that more than half of the patients had structural or functional abnormalities of the LV evaluated by echocardiography and almost one-fifth of the patients had undiagnosed HF. Previous studies have examined the distribution of HF stages in the community, but to our knowledge this is the first study to describe the prevalence of HF stages in elderly patients living with risk factors for HF, under relevant medical care. The findings of this study are in fine line with the prevalence of HF stages found in large community studies. ${ }^{4-6}$ However, by focusing on high-risk patients, we increased the observed prevalence of HF stages B and C. In the present study the patients in HF stage $\mathrm{C}$ were more likely to be older and have a history of AF, which was also the case for the patients in HF stage B. Despite symptoms of HF, no clinical characteristics clearly distinguished HF stages. Other studies have found an increased prevalence of higher age, male gender, obesity, atrial fibrillation and diabetes with higher stages of HF. ${ }^{40}$ Thus, the lack of differences in clinical characteristics between HF stages underscores the importance of focusing on symptoms in daily clinical practice in elderly patients with a risk factor for HF. Patients in HF stages A and B are considered patients at risk of overt HF. Previous studies have demonstrated an increased mortality risk and risk of admission for HF in patients in HF stage B. ${ }^{4-6}$ Further, it should be noticed that patients in HF stage B may have increased plasma concentrations of NT-proBNP and eventually signs of HF, but due to lack of symptoms these patients do not fulfil the criteria for overt HF (HF stage C). 


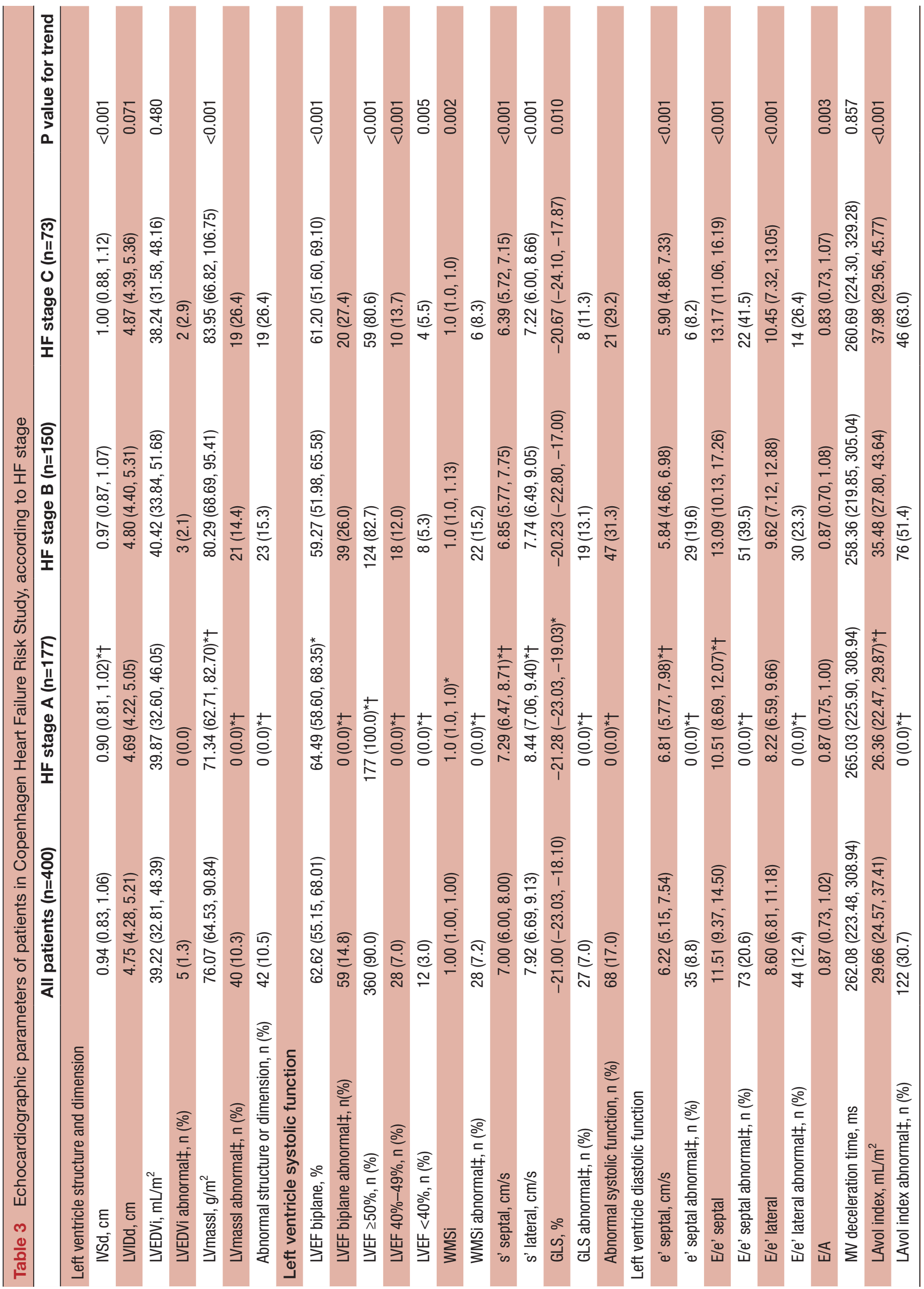

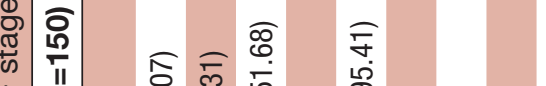

잉

产 ए।

के

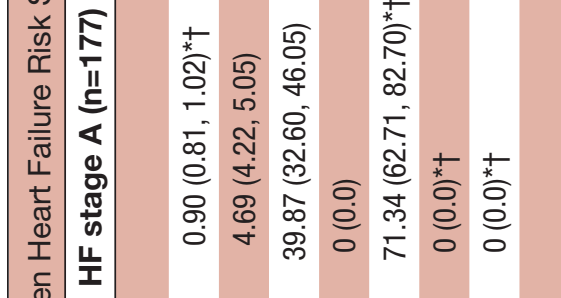

$\infty$

ठ + के



古 응

A 0 N 00 O

ธิ อ

s.

递 क

ช

ชิ
ळ N

N

일

$\stackrel{2}{2}$

$\stackrel{\text { 옹 }}{\overrightarrow{0}}$

잉

ํํㅇ

혹

킁

음

훙

$\frac{7}{3}$

ঠิ่

음.

กั

ก

స్

을

号

꿍 
Echocardiographic parameters were used to identify patients in both HF stages B and C. In this study comparable prevalence of structural and functional abnormalities of the myocardium was found in HF stages B and C, and only LV mass index and e' septal differed between HF stages B and C. Other studies have observed a more pronounced difference in echocardiographic variables, increasingly so with higher stages of $\mathrm{HF}^{4}{ }^{40}$

An important finding of this study was the substantial number of patients with HF stage $\mathrm{C}(\mathrm{n}=73)$, undiagnosed HF. Among patients with HF stage C, $80.6 \%$ had HFpEF (LVEF $\geq 50 \%$ ), $13.7 \%$ had HF with mid-range LVEF (LVEF $41 \%-50 \%$ ) and $5.5 \%$ had HF with reduced LVEF (LVEF $\leq 40 \%$ ). Compared with other studies the echocardiographic findings in this study confirm the increased risk of $\mathrm{HF}$ these patients present, with $\mathrm{E} / \mathrm{e}$ ' septal $>15$ in one-fifth of the patients ${ }^{21}$ and left atrial size $\geq 35 \mathrm{~mL} / \mathrm{m}^{2}$ in almost one-third of the patients. ${ }^{22}$ Finally, a total of 88 patients ( $n=69$ with sinus rhythm and $n=19$ with $A F$ ) had fulfilled the echocardiographic and NT-proBNP criteria for inclusion in the Paragon trial. However, the number was reduced to 27 when including the criteria of New York Heart Association (NYHA) class and treatment with a diuretic. ${ }^{23}$ Currently there is no established treatment for HFpEF; instead, focus on the treatment of comorbidities, for example, hypertension and atrial fibrilation, is recommended. In patients with type 2 diabetes, treatment with a sodium glucose inhibitor should be considered. ${ }^{24}$ Based on post-hoc analyses from the Irbesartan in Heart Failure With Preserved Systolic Function (I-PRESERVE) trial $^{25}$ and the Treatment of Preserved Cardiac Function Heart Failure with an Aldosterone Antagonist (TOPCAT) trial, ${ }^{26}$ it may be speculated that HF stage $\mathrm{C}$ patients, with relatively low plasma concentrations of NT-proBNP, could benefit from treatment with an aldosterone antagonist. ${ }^{27}$

\section{Cardiac biomarkers and HF stages}

Plasma concentrations of NT-proBNP increased with higher stages of HF. These observations are supported by similar findings in community-based studies of $\mathrm{HF}$ stages. ${ }^{467}$ Elevated plasma concentrations of NT-proBNP are expected in HF stage $\mathrm{C}$, but the elevated concentrations in HF stage B suggest that already in this early stage of HF, in asymptomatic patients, the heart is subject to an increased wall stress. This study is the first to describe that plasma concentrations of copeptin increase with increasing stages of HF. Copeptin is a surrogate marker for vasopressin and reflects neurohumoural activation. It has prognostic value in both acute and chronic HF, and in elderly patients with symptoms of $\mathrm{HF}^{28}$ We also observed increased plasma concentrations of MR-proADM with higher stages of HF, which also reflects increased neurohumoural activation in higher stages of HF. This finding has previously been observed in stable outpatients with $\mathrm{HF}^{29}$

In this present study, an increased proportion of patients in both HF stages $\mathrm{B}$ and $\mathrm{C}$ had detectable 
troponin-I, while other studies have found increasing plasma concentrations of troponin with each stage of HF. ${ }^{46}$ We found that only one-fifth of the patients had troponin-I above the detection limit, and from our observations elevated troponin-I is more coherent with an abnormal echocardiogram than with HF stages. Troponin-I has been associated with increased mortality risk. ${ }^{30}$

In contrast, higher stages of HF were not associated with increased plasma concentrations of hsCRP. Coronary microvascular inflammation has been suggested as a driver in the development of HFpEF. ${ }^{31}$ Lack of systemic inflammation or the need for a more sensitive biomarker reflecting inflammation ${ }^{32}$ may be the reason.

\section{Quality of life (MLHFQ) and Framingham risk score of HF}

Symptoms of HF were part of the definition of HF stage $\mathrm{C}$, and higher stages of HF more often presented with a higher NYHA class. Patients in HF stage C also presented with decreased quality of life compared with HF stages A and $\mathrm{B}$, respectively; this aspect has not been described in other studies. The quality of life decreases with severity of overt $\mathrm{HF}^{33}$ but it is noteworthy that also patients with undiagnosed $\mathrm{HF}$ stage $\mathrm{C}$ have a reduced quality of life.

In the present study Framingham 4-year risk score of congestive HF was increased in both HF stages B and C. However, the risk score was low, and the median probability for hospitalisation for congestion within 4 years was only increased by $3 \%$ for both men and women. Other studies have reported increased mortality rate with higher stages of HF, most pronounced for HF stages $\mathrm{C}$ and $\mathrm{D}^{4520}$ These numbers are hardly comparable since this study has no follow-up data; however, such a difference might occur due to different inclusion criteria.

\section{Methodological considerations and perspectives}

Some methodological considerations of this study should be addressed. Patients in this study were selected from three highly specialised sections at the hospital, according to predefined inclusion and exclusion criteria, with the purpose to evaluate whether HF was underdiagnosed in a high-risk population, with no current suspicion of $\mathrm{HF}$ by the treating clinician. Therefore, the findings in this cohort do not reflect the general population. Instead, as we intended, the study reflects the risk of HF in an elderly high-risk population.

The definition of abnormal echocardiographic parameters and the definition of HF in the present study need to be discussed. It may be argued that the definition of an abnormal echocardiography is too wide and that this has resulted in an overestimation of the prevalence of $\mathrm{HF}$ stages B and C. However, the definition was predefined before data analysis and inspired by current guidelines and previous studies. The ARIC study examined the effect of including diastolic measures in the definition of structural heart disease, and concluded that diastolic measures should be included ${ }^{4}$; similar results have been reported from Olmsted County. ${ }^{5}$ Further, the definition of overt HF (HF stage C) can also be discussed. We used the European Society of Cardiology's definition and required that symptoms of both dyspnoea or orthopnoea and oedema or use of loop diuretics were present. It was the intention to be conservative and avoid a type I error ('false positive cases'). In contrast, it may be argued that we have made a type II error ('false negative cases') because $30 \%$ of the patients in HF stage B were evaluated to be NYHA class II, but did not report symptoms themselves. A bicycle test with invasive measurement of filling pressures might have revealed reduced exercise capacity and increased filling pressures, thereby classifying these patients as HF stage C. ${ }^{34}$ Functional class was evaluated by a single investigator, and a stress test, for example, 6 min walking test, was not conducted in cases of doubt. Misclassification of functional status may therefore be present, but it should be noted that NT-proBNP increased according to HF stages. Dyspnoea may also be explained by ageing, obesity, physical fitness, chronic obstructive pulmonary disease and AF. Finally, we did not evaluate the prevalence of HF in different subgroups due to the sample size, and our data therefore elucidate the prevalence of early stages of $\mathrm{HF}$ in an elderly high-risk population with different risk factors for HF.

\section{CONCLUSION}

HF stages B and C are frequent in an elderly high-risk population without known or suspected HF, and especially HFpEF is underdiagnosed. Clinical characteristics are similar in early stages of HF, but the plasma concentrations of NT-proBNP are increasing with higher stages of $\mathrm{HF}$, and troponin-I is more frequently detectable if the echocardiography is abnormal. Finally, higher stages of $\mathrm{HF}$ are associated with a decreased quality of life even in patients with undiagnosed HF.

\section{Author affiliations}

${ }^{1}$ Department of Cardiology, Herlev and Gentofte University Hospital, Herlev, Denmark

${ }^{2}$ Faculty of Health and Medical Science, University of Copenhagen, Copenhagen, Denmark

${ }^{3}$ Department of Clinical Medicine, University of Copenhagen, Copenhagen, Denmark ${ }^{4}$ Department of Endocrinology, Rigshospitalet, Copenhagen, Denmark

${ }^{5}$ Department of Cardiology, Rigshospitalet, Copenhagen, Denmark

${ }^{6}$ Department of Internal Medicine KNEA, North Zealand University Hospital, Hillerod, Denmark

${ }^{7}$ Department of Nephrology, Herlev and Gentofte University Hospital, Herlev,

Denmark

${ }^{8}$ Department of Clinical Chemistry, Herlev and Gentofte University Hospital, Herlev, Denmark

${ }^{9}$ Department of Endocrinology, Herlev and Gentofte University Hospital, Herlev, Denmark

Contributorship statement FSG: conception and design, analysis and interpretation of data, drafting of the manuscript, agreement to be accountable for all aspects of the work in ensuring that questions related to the accuracy or integrity of any part of the work are appropriately investigated and resolved. CK, $\mathrm{CH}, \mathrm{LK}, \mathrm{PMH}, \mathrm{PRK}$, JF: conception and design, revising the manuscript critically for important intellectual content, final approval of the manuscript submitted. TK, NT, KKI: analysis and interpretation of data, revising the manuscript critically for important intellectual content, final approval of the manuscript submitted. MS: conception and design, analysis and interpretation of data, revising the manuscript critically for important intellectual content, agreement to be accountable for all 
aspects of the work in ensuring that questions related to the accuracy or integrity of any part of the work are appropriately investigated and resolved, final approval of the manuscript submitted.

Funding The conduct of this study was supported by research grants from The Danish Heart Foundation, Copenhagen, Denmark, and the TOYOTA foundation, Copenhagen, Denmark, and the Herlev and Gentofte University Hospital Research Council, Copenhagen, Denmark.

Competing interests None declared.

Patient consent for publication Parental/guardian consent obtained.

Ethics approval The study was approved by the Committee on Health Research Ethics for the Capital Region of Denmark (H-3-2014-016) and conducted according to the Declaration of Helsinki.

Provenance and peer review Not commissioned; externally peer reviewed.

Data sharing statement № additional data available.

Open access This is an open access article distributed in accordance with the Creative Commons Attribution Non Commercial (CC BY-NC 4.0) license, which permits others to distribute, remix, adapt, build upon this work non-commercially, and license their derivative works on different terms, provided the original work is properly cited, appropriate credit is given, any changes made indicated, and the use is non-commercial. See: http://creativecommons.org/licenses/by-nc/4.0/.

\section{REFERENCES}

1. Hunt SA, Baker DW, Chin MH. ACC / AHA practice guidelines ACC AHA guidelines for the evaluation and management of chronic heart failure in the adult: executive summary a report of the American college of cardiology / American heart association task force on practice guidelines. Circulation 2001;4636:2996-3007.

2. Vasan RS, Xanthakis V, Lyass A, et al. Epidemiology of Left Ventricular Systolic Dysfunction and Heart Failure in the Framingham Study: An Echocardiographic Study Over 3 Decades. JACC Cardiovasc Imaging 2018;11:1-10.

3. Ho JE, Lyass A, Lee DS, et al. Predictors of new-onset heart failure: differences in preserved versus reduced ejection fraction. Circ Heart Fail 2013;6:279-86.

4. Shah AM, Claggett B, Loehr LR, et al. Heart failure stages among older adults in the community: the Atherosclerosis Risk in Communities study. Circulation 2017;135:224-40.

5. Ammar KA, Jacobsen SJ, Mahoney DW, et al. Prevalence and prognostic significance of heart failure stages: application of the American College of Cardiology/American Heart Association heart failure staging criteria in the community. Circulation 2007; 115:1563-70.

6. Xanthakis V, Enserro DM, Larson MG, et al. Prevalence, Neurohormonal Correlates, and Prognosis of Heart Failure Stages in the Community. JACC: Heart Failure 2016;4:808-15.

7. McKie PM, Cataliotti A, Lahr BD, et al. The Prognostic Value of N-Terminal Pro-B-Type Natriuretic Peptide for Death and Cardiovascular Events in Healthy Normal and Stage A/B Heart Failure Subjects. J Am Coll Cardiol 2010;55:2140-7.

8. Alpert MA, Lavie CJ, Agrawal H, et al. Obesity and heart failure: epidemiology, pathophysiology, clinical manifestations, and management. Trans/ Res 2014;164:345-556.

9. Kannel WB, D'Agostino RB, Silbershatz $\mathrm{H}$, et al. Profile for estimating risk of heart failure. Arch Intern Med 1999;159:1197-204.

10. Lang RM, Badano LP, Mor-Avi V, et al. Recommendations for cardiac chamber quantification by echocardiography in adults: an update from the American Society of echocardiography and the European association of cardiovascular imaging. Eur Heart J Cardiovasc Imaging 2015;16:233-71.

11. Nagueh SF, Smiseth OA, Appleton CP, et al. Recommendations for the evaluation of left ventricular diastolic function by echocardiography: an update from the American society of echocardiography and the European association of cardiovascular Imaging. J Am Soc Echocardiogr 2016;29:277-314.

12. Cheng S, Larson MG, McCabe EL, et al. Age- and sex-based reference limits and clinical correlates of myocardial strain and synchrony: the Framingham Heart study. Circ Cardiovasc Imaging 2013;6:692-9.

14. Ponikowski P, Voors AA, Anker SD, et al. 2016 ESC guidelines for the diagnosis and treatment of acute and chronic heart failure: the task Force for the diagnosis and treatment of acute and chronic heart failure of the European Society of cardiology (ESC). developed with the special contribution of the heart Failure Association (HFA) of the ESC. Eur J Heart Fail 2016;18:891-975.

15. Shelton RJet al. The diagnostic utility of N-terminal pro-B-type natriuretic peptide for the detection of major structural heart disease in patients with atrial fibrillation. Eur Heart J 2006;27:2353-61.

16. Yeo KT, Wu AH, Apple FS, et al. Multicenter evaluation of the Roche NT-proBNP assay and comparison to the biosite triage BNP assay. Clin Chim Acta 2003;338(1-2):107-15.

17. Caruhel P, Mazier C, Kunde J, et al. Homogeneous timeresolved fluoroimmunoassay for the measurement of midregional proadrenomedullin in plasma on the fully automated system B.R.A.H.M.S KRYPTOR®. Clin Biochem 2009;42(7-8):725-8.

18. Morgenthaler NG. Assay for the measurement of copeptin, a stable peptide derived from the precursor of vasopressin. Clin Chem 2006;52:112-9.

19. Casals G, Filella X, Bedini JL. Evaluation of a new ultrasensitive assay for cardiac troponin I. Clin Biochem 2007;40:1406-13.

20. Yang $\mathrm{H}$, Negishi $\mathrm{K}$, Wang $\mathrm{Y}$, et al. Echocardiographic screening for non-ischaemic stage $B$ heart failure in the community. Eur $J$ Heart Fail 2016:18:1331-9.

21. Nagueh SF, Middleton KJ, Kopelen HA, et al. Doppler tissue imaging: a noninvasive technique for evaluation of left ventricular relaxation and estimation of filling pressures. J Am Coll Cardiol 1997;30:1527-33.

22. Møller JE, Hillis GS, Oh JK, et al. Left atrial volume: a powerful predictor of survival after acute myocardial infarction. Circulation 2003;107:2207-12.

23. Solomon SD, Rizkala AR, Gong J, et al. Angiotensin Receptor Neprilysin Inhibition in Heart Failure With Preserved Ejection Fraction: Rationale and Design of the PARAGON-HF Trial. JACC Hear Fail 2017:5:471-82.

24. Fitchett $\mathrm{D}$, Butler J, van de Borne $\mathrm{P}$, et al. Effects of empagliflozin on risk for cardiovascular death and heart failure hospitalization across the spectrum of heart failure risk in the EMPA-REG OUTCOME $®$ trial. Eur Heart J 2018;39:363-70.

25. Anand IS, Rector TS, Cleland JG. Prognostic value of baseline plasma amino-terminal pro-brain natriuretic peptide and its interactions with irbesartan treatment effects in patients with heart failure and preserved ejection fraction. Circ: Heart Fail 2011;4:569-77.

26. Anand IS, Claggett B, Liu J, et al. Interaction Between Spironolactone and Natriuretic Peptides in Patients With Heart Failure and Preserved Ejection Fraction. JACC: Heart Fail 2017;5:241-52.

27. Pfeffer MA, Claggett B, Assmann SF, et al. Regional variation in patients and outcomes in the treatment of preserved cardiac function heart failure with an aldosterone antagonist (TOPCAT) trial. Circulation 2015;131:34-42.

28. Alehagen Uet al. Association of copeptin and $\mathrm{N}$-terminal proBNP concentrations with risk of cardiovascular death in older patients with symptoms of heart failure. JAMA 2011;305:2088-95.

29. Xue Y, Taub P, lqbal N, et al. Mid-region pro-adrenomedullin adds predictive value to clinical predictors and Framingham risk score for long-term mortality in stable outpatients with heart failure. Eur $J$ Heart Fail 2013;15:1343-9.

30. de Lemos JA, Drazner MH, Omland T, et al. Association of Troponin T Detected With a Highly Sensitive Assay and Cardiac Structure and Mortality Risk in the General Population. JAMA 2010;304:2503.

31. Paulus WJ, Tschöpe C. A novel paradigm for heart failure with preserved ejection fraction: comorbidities drive myocardial dysfunction and remodeling through coronary microvascular endothelial inflammation. J Am Coll Cardiol 2013;62:263-71.

32. Christensen HM, Frystyk J, Faber J, et al. $\alpha$-Defensins and outcome in patients with chronic heart failure. Eur $\mathrm{J}$ Heart Fail 2012;14:387-94.

33. Rector TS, Cohn JN. Assessment of patient outcome with the Minnesota Living with Heart Failure questionnaire: reliability and validity during a randomized, double-blind, placebo-controlled trial of pimobendan. Pimobendan Multicenter Research Group 1992.

34. Andersen MJ, Ersbøll M, Bro-Jeppesen J, et al. Exercise hemodynamics in patients with and without diastolic dysfunction and preserved ejection fraction after myocardial infarction. Circ Heart Fail 2012;5:444-51.

35. Gaborit F, Kistorp C, Kumler T, 2018. Prevalence of early stages of heart failure in an elderly high risk population: the copenhagen heart failure risk study. ESC365. Available from: https://esc365.escardio. org/Congress/Heart-Failure-2018-World-Congress-on-AcuteHeart-Failure/Moderated-Poster-session-2-Epidemiology/172475prevalence-of-early-stages-of-heart-failure-in-an-elderly-high-riskpopulation-the-copenhagen-heart-failure-risk-s 